\title{
Terapias de masaje para la profilaxis de la lesión Epicondilitis lateral del codo ligada a la práctica deportiva de atletas de tenis de mesa Paralimpico de la academia Provincial Mayabeque
}

Massage therapies for the prophylaxis of the side epicondylitis injury of the elbow linked to the sports practice of Paralympic table tennis athletes of the

Provincial Mayabeque academy

MSc. Eugenio Rodolfo González Pérez. ${ }^{1}$

Recibido: 07-10-2018 / Revisado: 09-11-2018 / Aceptado: 11-12-2018 / Publicado: 04-01-2019

\section{https://doi.org/10.33262/anatomiadigital.v2i1.1056}

\begin{abstract}
.
This work deals with the prophylaxis of the side epicondylitis lesion of the elbow, a topic of great importance in Paralyctable table tennis of the Provincial Mayabeque academy. Our goal is to select massage therapies for the prophylaxis of the side epicondylititis lesion of the elbow linked to the sports practice of table tennis athletes, ACLIFIM and ANSOC. This research allows us to work the prophylaxis of the injury from massage therapies which will have as fundamental elements the therapeutic massage and points to be treated with digitopuntura. For the elaboration of this work we rely on the review
\end{abstract}

\section{Resumen.}

Este trabajo trata sobre la profilaxis de la lesión Epicondilitis lateral del codo, un tema de gran importancia en tenis de mesa Paralimpico de la academia Provincial Mayabeque. Nuestro objetivo es seleccionar terapias de masaje para la profilaxis de la lesión Epicondilitis lateral del codo ligada a la práctica deportiva de atletas de Tenis de Mesa, de ACLIFIM y ANSOC. Esta investigación nos permite trabajar la profilaxis de la lesión a partir de terapias de masaje la cual tendrá como elementos fundamentales el masaje terapéutico y puntos a tratar con digitopuntura. Para la elaboración

\footnotetext{
${ }^{1}$ Ciencias Médicas Mayabeque, Departamento Cultura Física jefe de Departamento, Mayabeque, Cuba, eugeniogp@eiefd.co.cu iD https://orcid.org/0000-0002-7353-0151
} 
of the literature related to the subject, the observation of the training process and participation in the national arbitration course. Table tennis classification with the aim of obtaining information about the occurrence of such injury and actions that are performed during the training for the prophylaxis of the same, the individual survey of trainers and Arbitrators classifiers in order to know the most common injury during the training process and the pathways for prophylaxis of it and the statisticalmathematical method that allowed us to perform the percentage calculation of the behavior of all the incidents that occurred during the observation and the survey. With this work we hope to achieve better physical and psychic harmony on the part of practitioners so therapeutic massage therapies and digitopuntura will largely help to acquire a good physical conditioning and in this way decrease gradually cases of sideelbow epicondylitis injury in the Mesa Tennis team of the ACLIFIM and ANSOC of the Mayabeque Provincial

Keywords: Therapeutic massage, sports injuries de este trabajo nos apoyamos en la revisión de la bibliografía relacionada con el tema, la observación al proceso de entrenamiento y en la participación en el curso nacional de arbitraje. Clasificación de tenis de mesa con el objetivo de obtener información acerca de la ocurrencia de dicha lesión y acciones que se realizan durante el entrenamiento para la profilaxis de la misma, la encuesta individual a entrenadores y Arbitros clasificadores con el fin de conocer la lesión más frecuente durante el proceso de entrenamiento y las vías para la profilaxis de la misma y el método estadístico-matemático que nos permitió realizar el cálculo porcentual del comportamiento de todas las incidencias que se presentaron durante la observación y la encuesta. Con este trabajo esperamos lograr una mejor armonía física y psíquica por parte de los practicantes por lo que las terapias de masaje terapéutico y digitopuntura ayudaran en gran parte a adquirir un buen acondicionamiento físico y de esta manera disminuir paulatinamente los casos de lesionados por Epicondilitis lateral de codo en el equipo de Tenis de Mesa de la ACLIFIM y ANSOC de la Provincial Mayabeque

Palabras claves: Masaje terapéutico, lesiones deportivas

\section{Introducción.}

Biriukov, (2003), Conjunto de manipulaciones que debidamente dosificadas ejercen una acción mecánica y refleja sobre los tejidos y órganos. Técnica fisioterapéutica preferiblemente manual o mecánica con el objetivo de manipular, movilizar, sedar o estimular la piel, músculos, ligamentos, tendones, articulaciones y vísceras con efectos locales y sistémicos. 
Hernández (2003) lo define como una "experiencia relajante, donde sus efectos no solo son a nivel físico, la mente también responde al toque humano."

"Las lesiones deportivas son aquellas alteraciones de los huesos, articulaciones, músculos y tendones que se producen durante la práctica de actividades físicas y se hallan relacionadas con el gasto deportivo". (Caridad Rodríguez, 2001).

"Es tiempo que el deportista este alejado de la competición (tiempo perdido) como el determinante principal.”(Ronald. P. Pffeifer, 2000).

\section{Desarrollo}

\section{Lesiones más frecuentes en el Tenis de Mesa.}

"Dentro del conjunto general de las llamadas Alopatías, el Tenis de Mesa es una actividad deportiva con poco índice de accidentes, las alteraciones se deben más a desórdenes de índole técnico que cualquier otra cosa". (Méndez, 1999).

Pero como todo deporte si se practica en un ambiente competitivo acarrea lesiones. En este las que se presentan mayormente son tendinitis del hombro (hombro del tenista), lesiones de la rodilla, distensión de los gemelos, ruptura del tendón de Aquiles, esguinces de tobillo.

Un gran capítulo, por lo frecuente, incapacitante y de difícil tratamiento son las patologías del codo.

Otras disciplinas deportivas lo tienen como causa frecuente de enfermedad (Béisbol, Fútbol, Lucha, Boxeo, Golf, Tenis de Campo, Tenis de Mesa).

Entre las lesiones más frecuentes en el Codo de tenista hablaremos con detenimiento de las EPICONDALGIAS, ya que es la que representa el porcentaje más elevado de los padecimientos.

Aquí entran en consideración todas las causas favorecedoras de patología crónica de inserción.

- $\quad$ Errores de adiestramiento.

- Técnicas defectuosas.

- Progresión mal conducida.

- Falta de estiramiento pre y post actividad deportiva.

- Falta de calentamiento. 
- Material inadecuado.

- Excesos cuantitativos.

- Focos de infección a distancia.

A continuación, planteamos los tres síndromes que pueden ocurrir dentro de las patologías del codo asociadas a la práctica deportiva

1. Enfermedad de inserción epicondilea. Epicondilitis lateral Codo de tenista Existencia de molestias subjetivas como dolores en la práctica deportiva y al realizar ciertos movimientos de la vida diaria (Abrir una puerta, llenar un vaso con la botella llena, apretar tornillos, etc.

2. Síndrome intraarticular: Puede sospecharse únicamente mediante una palpación precisa. Se provoca dolor a la presión digital de la cabeza radial o del condilo.

3. Síndrome neurógeno. Corresponde a una neuralgia de la rama posterior del nervio radial. Se trata de una Epicondalgia que llama la atención por algunos caracteres distintivos: Rebeldía al tratamiento. La exacerbación Paroxística Nocturna. Y la casi nula significación al examen por palpitación. el electromiograma revela de forma inconstante signos de déficit neurógeno de la rama posterior del nervio radial.

Los tres síndromes pueden ocurrir aisladamente o asociadas en un mismo individuo.

Objetivo de la Investigación: Seleccionar un conjunto de terapias de masaje para la profilaxis de la Epicondilitis lateral del codo ligada a la práctica deportiva de atletas de Tenis de Mesa de la Aclifim y Asoc. del área deportiva Carlos Baliño del municipio Güines.

\section{Materiales y Métodos}

- Métodos teóricos: Histórico-lógico, inducción-deducción, análisis-síntesis, enfoque sistémico y los Métodos empíricos: revisión de documentos, observación

Tabla 1 Resultados de la Observación

\begin{tabular}{|c|c|}
\hline $\begin{array}{l}\text { Resultados } \\
\text { de la }\end{array}$ & $\begin{array}{l}\text { De los } 6 \text { atletas que se lesionan del } \\
\text { codo durante la parte principal de la } \\
\text { clase todos corresponden a la } \\
\text { Epicondilitis lateral del codo. Según }\end{array}$ \\
\hline onservacion & $\begin{array}{l}\text { característicos de la lesión } \\
\text { observados. }\end{array}$ \\
\hline
\end{tabular}


Vol. 2, $\mathrm{N}^{\circ} 1$, p. 28-38, enero-marzo, 2019

\begin{tabular}{|c|c|c|c|} 
Síntomas & $\begin{array}{c}\text { Dolor en la } \\
\text { parte } \\
\text { externa del } \\
\text { codo }\end{array}$ & $\begin{array}{c}\text { Dolor al } \\
\text { enderezar } \\
\text { o levantar } \\
\text { la muñeca }\end{array}$ & $\begin{array}{c}\text { Dolor que } \\
\text { se hizo } \\
\text { más agudo } \\
\text { cuando } \\
\text { levantaban } \\
\text { un objeto } \\
\text { pesado }\end{array}$ \\
\hline Resultados & $50 \%$ & $33,30 \%$ & $16,60 \%$ \\
\hline $\begin{array}{l}\text { Los entrenadores no emplean ningún procedimiento } \\
\text { que le permita la profilaxis de esta lesión, sin } \\
\text { embargo, los entrenadores al detectar estos síntomas } \\
\text { detienen la realización del ejercicio e indican al } \\
\text { atleta que debe tratarse con el médico. }\end{array}$ \\
\hline
\end{tabular}

Fuente: Elaboración propia.

Tomando como base todo lo anteriormente planteado Seleccionamos un conjunto de terapias de masaje, la cual tendrá como elementos fundamentales el masaje terapéutico y puntos a tratar con digitopuntura para la profilaxis de la Epicondilitis lateral del codo en los tenistas.

Para trabajar la digitopuntura primero hay que conocer la localización de cada uno de los puntos a localizar, así como su metodología.

La medida de distancias anatómicas a que se refieren la descripción para la localización de los puntos es el de unidad relativa del cuerpo, que en chino se llama "Cun" y que se pronuncia "tsun.

Un cun es el grueso del dedo pulgar de la persona a quien se le trata. El ancho de tres pulgares es lo mismo que cuatro dedos de la mano excluyendo el pulgar. Una y media unidad, por tanto, puede ser medida por el ancho de dos dedos adyacentes (excluyendo el pulgar). (Ver anexo 9). Los puntos de acupuntura son sensibles a la presión porque esos puntos forman un sistema de alarma de dos vías. Cuando duele una parte del cuerpo -la cabeza, por ejemplo- al punto correspondiente (en este caso es el punto 1 entre el pulgar y el índice) será muy sensitivo porque el reflejo se ha alarmado. Por otro lado, la estimulación de ese punto envía un mensaje a la base del cerebro quien inicia la acción curativa apropiada. Una forma que el acupunturista usa para saber qué punto seleccionar y su exacta localización es presionando el punto que él cree que necesita tratamiento. Si este es sensible, es que está en el punto y localización correcta y probablemente producirá efectos terapéuticos. Los puntos más efectivos, son los que están más lejos del órgano afectado y son muy sensibles cuando se presionan. La selección del punto tiene que corresponderse a sus síntomas, luego ver la localización del punto y buscar el área donde duele más. Cuando se alcanza el punto más 
sensible ya se sabe que lo ha encontrado. Luego para tratar se aplica un poco de presión hasta que surja un poco de dolor en este punto.

Objetivo:

Fortalecer los grupos musculares y los componentes articulares del codo a través de un conjunto de terapias de masaje.

Procedimiento:

- Para la digito presión: Individual y sentados.

- Para el masaje en parejas. El que recibe el tratamiento acostado decúbito prono.

Metodología

- Para el masaje

- Se aplicará en la parte inicial.

- Se aplicará una fricción concéntrica y una frotación en forma de pinza durante 2 minutos.

- La fricción concéntrica la cual se ejecuta con ambas manos con movimientos circulares en forma de número 8. Con los pulgares se fricciona una zona de la articulación, mientras que con los demás dedos la otra.

- La frotación en forma de pinza se realizará con una mano en forma de arco sobre la articulación desplazándose hacia arriba. Con el pulgar se frota una parte de la articulación y con los demás dedos la otra parte.

- Los atletas permanecerán 10 min. en reposo, este tiempo es para que el masaje sea efectivo.

Para la digitopuntura.

Se realizará la localización de los siguientes puntos.

- Vesícula Biliar 34 (VB34). Localización: Situado por debajo y por delante de la cabeza de la fíbula. Punto de influencia para ligamentos y tendones. - Trastornos musculares, de ligamentos y tendones; dolor de la cadera, rodilla y tobillo, ciatalgia; espasmos musculares del miembro inferior. 
- Triple Recalentador (TR5). Localización: Región dorsal del antebrazo, a 2 cun por encima del pliegue de la articulación radiocarpiana, entre el radio y la uña. Cefalea lateral, dolor de la mejilla; dolor del oído, sordera, tinnitus; dolor y trastornos motores del hombro, brazo, codo y dedos.

- Pulmón cinco (P5). Localización: Con el antebrazo ligeramente flexionado sobre el brazo, se encuentra a nivel del pliegue del codo, cerca del borde radial del tendón de inserción del músculo. Punto de dispersión. - Tos, disnea, opresión torácica, dolor de garganta; dolor del codo y del brazo.

- Corazón siete (C7). Localización: Con el brazo en supinación. Está situado en la región anterior del antebrazo, en una depresión en el lado radial del tendón del músculo flexor ulnar del carpo, a nivel del pliegue transverso de la articulación radiocarpiana por detrás del hueso pisiforme. Importante punto sedante. - Dolor precordial, ansiedad, palpitaciones; irritabilidad, histeria, insomnio.

- Intestino grueso 11 (IG11). Localización: Con el antebrazo ligeramente flexionado sobre el brazo, se encuentra a nivel del pliegue del codo, cerca del borde radial del tendón de inserción del músculo. Punto tonificante, homeostático, estimulante de los factores antipatógenos, antinflamatorio, hipotermizante. - Dolor del codo, del brazo, del hombro; alergia, urticaria, fiebre.

- Intestino grueso Cuatro (IG4). Localización: Cara dorsal de la mano, entre el 1ro. Y 2do. Metacarpiano. Extendiendo los dedos pulgar e índice y uniéndolos estrechamente se encuentra en el punto más alto de la prominencia que se forma. Punto más analgésico, punto distal de la parte anterior de la cabeza y el cuello. Cefalea frontal, dolor de garganta, de los ojos, de los dientes; alergia, fiebre; dolor del hombro, del brazo, calambres de la mano; dolor abdominal.

Se aplicará presión digital simple con el método de armonización, 10 segundos hacia la derecha y 10 segundos hacia la izquierda en cada punto durante 40 segundos.

Se harán 5 repeticiones en cada punto.

Indicaciones Metodológicas.

- El atleta debe tener la extremidad totalmente relajada.

Para el masaje.

- El atleta debe estar acostado de cúbito supino.

- Se aplica la fricción antes y después la frotación. 
- Culminado el masaje se reposa $10 \mathrm{~min}$.

- El atleta que recibe el masaje no debe tener contraindicaciones.

- Las sesiones se inician con poca intensidad y profundidad para ir logrando la adaptación del organismo del atleta.

- La frotación tiene que ser suave y profunda.

Para la digito presión:

- Se marcan cada uno de los puntos antes de comenzar para que no exista pérdida del tiempo.

- En digito presión se utiliza para la armonización la punta de los dedos pulgares y del medio.

- La presión se realizará incrementando gradualmente la fuerza efectuando movimientos circulares a ambos lados de forma rápida hasta que el atleta sienta una sensación de entumecimiento y dolor sordo.

- Para terminar, se hace decrecer suavemente la presión.

Evaluación: Se medirán por los parámetros de la aparición de los síntomas y signos clínicos de la epicondilitis.

- Dolor al enderezar o levantar la muñeca y la mano.

- Dolor en reposo de la muñeca y la mano.

- Sensibilidad a la palpación del epicondilo lateral.

- Dolor o sensibilidad en la parte externa del codo.

- Dolor que comienza en el codo y se invada en el antebrazo o parte superior del brazo.

- Debilidad de la musculatura de la muñeca.

La evaluación del sistema se propone que sea:

1. Muy eficaz: Inferior a la media más dos veces la desviación estándar.

2. Eficaz: De la media (x) más la desviación estándar hasta la media más dos veces la desviación estándar. 
3. Poco eficaz: De la media más 0,5 de la desviación hasta la media más una desviación estándar.

En esta evaluación se sabrá si el tratamiento que se está proponiendo puede ser o no utilizado con respecto a los resultados que se obtendrán después de aplicado, es decir, si luego de un año de emplearlo se observa que más de la mitad de la muestra no ha presentado ninguno de los parámetros, por los que está enmarcada la lesión, nos está simplemente confirmando la utilidad del tratamiento como también puede demostrar lo contrario y se tendrá que ver qué fue lo que falló.

\section{Conclusiones.}

- Los fundamentos teóricos sobre el tratamiento de las lesiones en los atletas de Tenis de Mesa nos permitieron darle el basamento científico necesario a nuestra investigación para evitar que los tenistas de nuestro municipio tengan efectos negativos en las actividades que realizan.

- La lesión más frecuente que padecen los tenistas de nuestro municipio es la epicondilitis lateral del codo, producida en la parte principal de la clase en un $100 \%$.

- La selección del conjunto de terapias de masaje les permitirá a los entrenadores incidir directamente en la profilaxis de esta lesión, en su área de trabajo, favoreciendo así los logros deportivos del área de tenis de mesa del municipio.

\section{Referencias bibliográficas.}

Bobath, B. y K.Bobath (1991). Desarrollo motor en distintos tipos de Parálisis Cerebral. Buenos Aires, Editorial Médica Panamericana.

Bobath, K. (1982) Base neurofisiológica para el tratamiento de la Parálisis Cerebral. Buenos Aires, Editorial Médica Panamericana.

Estévez Cullell, M. y col. (2004). La Investigación Científica en la Actividad Física: su Metodología. Ciudad de la Habana, Editorial Deportes.

Fernández Nieves Y. (2007) Prevención de las Necesidades Educativas Especiales (NEE) Editorial Deportes. Ciudad de la Habana. Cuba

Genot C. y col. (1988). Kinesioterapia I, II, III, IV. Buenos Aires, Editorial MédicaPanamericana.

Krussen Hammond, Frank. (1992). Medicina Física y Rehabilitación. Buenos Aires, Editorial Panamericana 
López Chicharro, José y col. (2002). El desarrollo y el rendimiento deportivo. Madrid, Editorial Gymnos

Manual de Merck. Disponible en: http:// www. msd.es / mmerck / m201. Html Consultado el 15 de Febrero de 2011

Popov, S. N. (1988). La cultura física terapéutica. Moscú, Ráduga,

Rodríguez Santana, Juliet (2005). La equinoterapia en el manejo rehabilitador de la parálisis cerebral en niños de 4 a 12 años pertenecientes al CIS "La Pradera". Trabajo de Diploma. Ciudad de la Habana, ISCF "Manuel Fajardo".

Sentmanat Belisón, Armando. (1998). Neurorehabilitación Multifactorial Intensiva. Ciudad de la Habana, ACM Actualización en las Ciencias Médicas.

Sociedad Española de Rehabilitación y Medicina Física. (1996). Rehabilitación. Volumen 30. Fascículo 6. Revista de la Sociedad Española de Rehabilitación y Medicina Física. Madrid.

Trujillo Aldama, Ligia y col. (1997). Fundamentos de defectología. Ciudad de la Habana, Editorial Pueblo y Educación.

Valdés Casal, Hiram y col. (1987). Introducción a la investigación científica aplicada a la Educación Física y el Deporte. Ciudad de la Habana, Editorial Pueblo y Educación.

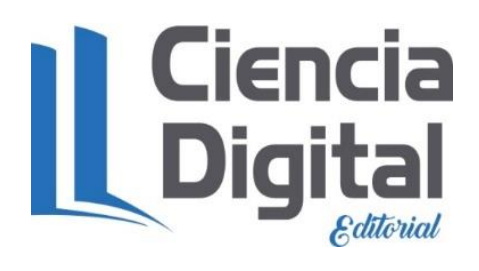




\section{PARA CITAR EL ARTÍCULO INDEXADO.}

González Pérez, E. R. (2019). Terapias de masaje para la profilaxis de la lesión Epicondilitis lateral del codo ligada a la práctica deportiva de atletas de tenis de mesa Paralimpico de la academia Provincial Mayabeque. Anatomía Digital, 2(1), 28-38. https://doi.org/10.33262/anatomiadigital.v2i1.1056

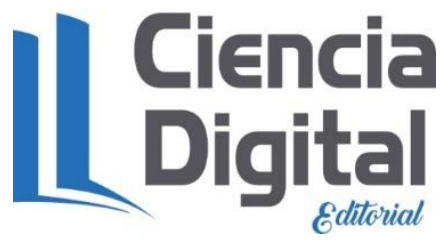

El artículo que se publica es de exclusiva responsabilidad de los autores y no necesariamente reflejan el pensamiento de la Revista Anatomía Digital.

El artículo queda en propiedad de la revista y, por tanto, su publicación parcial y/o total en otro medio tiene que ser autorizado por el director de la Revista Anatomía Digital.
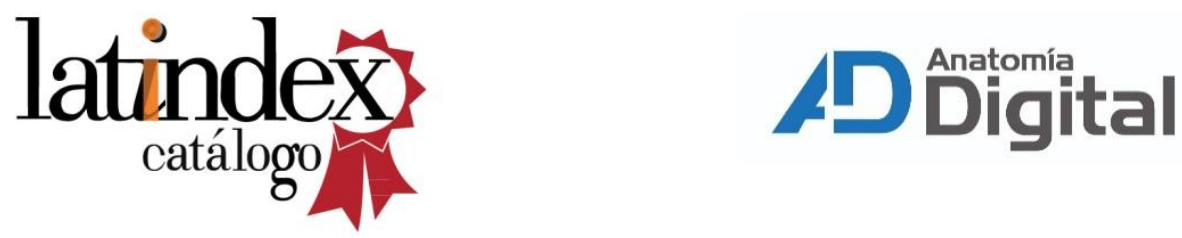